\title{
Active Galactic Nuclei Jets as the Origin of Ultrahigh-Energy Cosmic Rays and Perspectives for the Detection of Astrophysical Source Neutrinos at EeV Energies
}

\author{
Xavier Rodrigues®, ${ }^{*}$ Jonas Heinze, Andrea Palladino, Arjen van Vliet@, and Walter Winter® \\ DESY, Platanenallee 6, 15738 Zeuthen, Germany
}

(Received 25 March 2020; revised 21 October 2020; accepted 1 April 2021; published 10 May 2021)

\begin{abstract}
We demonstrate that a population of active galactic nuclei (AGN) can describe the observed spectrum of ultra-high-energy cosmic rays (UHECRs) at and above the ankle, and that the dominant contribution comes from low-luminosity BL Lacertae objects. An additional, subdominant contribution from high-luminosity AGN is needed to improve the description of the composition observables, leading to a substantial neutrino flux that peaks at exaelectronvolt $(\mathrm{EeV})$ energies. We also find that different properties for the low- and high-luminosity AGN populations are required; a possibly similar baryonic loading can already be excluded from current IceCube Neutrino Observatory observations. We also show that the flux of neutrinos emitted from within the sources should outshine the cosmogenic neutrinos produced during the propagation of UHECRs. This result has profound implications for the ultra-high-energy ( $\sim \mathrm{EeV})$ neutrino experiments, since additional search strategies can be used for source neutrinos compared to cosmogenic neutrinos, such as stacking searches, flare analyses, and multimessenger follow-ups.
\end{abstract}

DOI: 10.1103/PhysRevLett.126.191101

Blazars are active galactic nuclei (AGN) with their jets pointed toward Earth; they contribute more than $80 \%$ of the extragalactic $\gamma$-ray background [1], dominating the $\gamma$-ray emission above $50 \mathrm{GeV}$. In addition, there are strong indications for correlations between the arrival directions of ultra-high-energy cosmic rays (UHECRs) and extragalactic $\gamma$-ray sources, including AGN [2]. Jetted AGN are also one of the candidate source classes which may have sufficient power to maintain the UHECR flux. As a consequence, it is natural to consider jetted AGN as the possible origin of the observed UHECRs.

In addition, a diffuse flux of high-energy astrophysical neutrinos has been discovered [3]. This may be a direct indicator for the origin of UHECRs because neutrinos point back directly to their sources, while UHECRs are deflected by Galactic and extragalactic magnetic fields. The recent detection of neutrinos from the flaring blazar TXS $0506+$ 056 provides further evidence that cosmic rays are accelerated in AGN up to at least petaelectronvolt $(\sim \mathrm{PeV})$ energies [4,5], see also earlier results [6]. On the other hand, dedicated catalog searches for neutrinos from known AGN limit the contribution of these objects to about $20 \%$ of the diffuse IceCube flux [7], which means that resolved $\gamma$-ray blazars are probably not the dominant source of neutrinos at teraelectronvolt-petaelectronvolt (TeV-PeV) energies. However, low-luminosity or high-redshift AGN are more numerous and less constrained observationally, and may still, under certain conditions, be a viable candidate for the diffuse IceCube neutrino flux [8,9].

On the other hand, if AGN significantly contribute to the observed UHECR flux, they need to accelerate cosmic-ray nuclei up to $\sim 10^{20} \mathrm{eV}$. The production of UHECRs of these extreme energies in AGN is supported by studies involving the simulation of different reacceleration mechanisms [10-12]. Photointeractions of these cosmic rays lead to the emission of neutrinos with energies $E_{\nu} \simeq 0.05 E_{\mathrm{CR}} / A$, where $A$ is the mass number of the cosmic-ray nucleus. AGN would then be expected to yield significant neutrino fluxes in the exaelectronvolt $(\mathrm{EeV})$ energy range, where currently only upper limits exist $[13,14]$.

The production of UHECRs and neutrinos in AGN has been studied in previous works [e.g., [15-28] ]. Other more phenomenology-driven works have had the objective of describing the observed UHECR spectrum and composition with a population of high-energy sources [29-31]. However, a self-consistent description of the UHECR spectrum and composition including a neutrino flux prediction from the entire AGN population has not yet been performed.

The objective of this study is twofold: (a) to investigate under what conditions the AGN population can explain the UHECR spectrum observed by the Pierre Auger Observatory (henceforth, Auger) [32], while obeying the most recent IceCube Neutrino Observatory limits at $\sim \mathrm{PeV}$ energies [7]; and (b) to investigate the corresponding neutrino fluxes, particularly in the $\mathrm{EeV}$ range. This includes both source neutrinos as well as cosmogenic, i.e., those produced in UHECR interactions during their propagation over extragalactic distances.

Cosmogenic neutrinos are the main target of radiodetection neutrino experiments in the $\mathrm{EeV}$ range such as the radio array of IceCube-Gen2 [33], GRAND [34], ARA [35], and ARIANNA [36]. Recent descriptions of the UHECR spectrum and composition, however, indicate that the maximum energies are limited by the accelerators, and 
that they can be described by a rigidity dependence $E_{\mathrm{CR}} \propto Z$, with $Z$ the charge of the nucleus [37]. Such a rigidity dependence is generated, for instance, if the particles are magnetically confined to a certain zone of fixed size. This framework leads to low cosmogenic neutrino fluxes $[38,39]$, except for a potential contribution from a subdominant proton population [40]. By considering AGN as the sources of the UHECRs, we will actually reevaluate the hypothesis that the $\mathrm{EeV}$ neutrino sky is dominated by cosmogenic neutrinos, and we will show that source neutrinos may actually be the foreground signal at these energies.

We assume that cosmic rays are reaccelerated in AGN jets to a power-law spectrum up to ultrahigh energies. We then utilize a combined source-propagation model, which has essentially three components (see Supplemental Material [41] for details): (1) simulation of the photohadronic interactions that the accelerated UHECRs undergo in a radiation zone in the jet. This step is relevant in high-luminosity sources, where these interactions lead to efficient neutrino emission and the production of a nuclear cascade, which is simulated self-consistently. (2) Propagation of the escaping cosmic rays toward Earth. This also involves the numerical calculation of photointeractions, including the development of a nuclear cascade and the production of cosmogenic neutrinos. (3) Extension of the calculation to the entire cosmological distribution of AGN. The resulting overall UHECR and neutrino fluxes are then compared to current measurements.

We divide AGN into three subpopulations based on their different cosmological evolution functions (cf. Fig. 1): lowluminosity BL Lacertae objects (BL Lacs), high-luminosity BL Lacs, and flat-spectrum radio quasars (FSRQs); we refer to the latter two categories as "high-luminosity AGN." While blazars emit highly beamed $\gamma$ rays and neutrinos in the direction of Earth, the observed UHECRs can originate in the broader family of jetted AGN, since these particles are deflected in magnetic fields. We assume that the cosmic evolution of blazars is representative of this overall AGN population, in view of the unified scheme of radio-loud AGN [64]. We adopt the cosmological evolution model illustrated in Fig. 1 as a function of redshift and $\gamma$-ray luminosity for these AGN subclasses [65,66]. This evolution model yields a total $\gamma$-ray emissivity from AGN that is consistent with the diffuse Fermi Large Area Telescope (Fermi LAT) $\gamma$-ray measurements [67]. Our starting hypothesis is that these AGN populations have similar properties regarding the UHECR acceleration; we will demonstrate, however, that at least the baryonic loading (i.e., the ratio between the amount of energy injected into cosmic rays and that injected into electrons) has to differ for low- and high-luminosity sources in order to satisfy $\mathrm{PeV}$ neutrino constraints.

Regarding the chemical injection composition of cosmic rays, we consider a mixture of four representative mass groups: protons, helium-4, nitrogen-14, and iron-56.
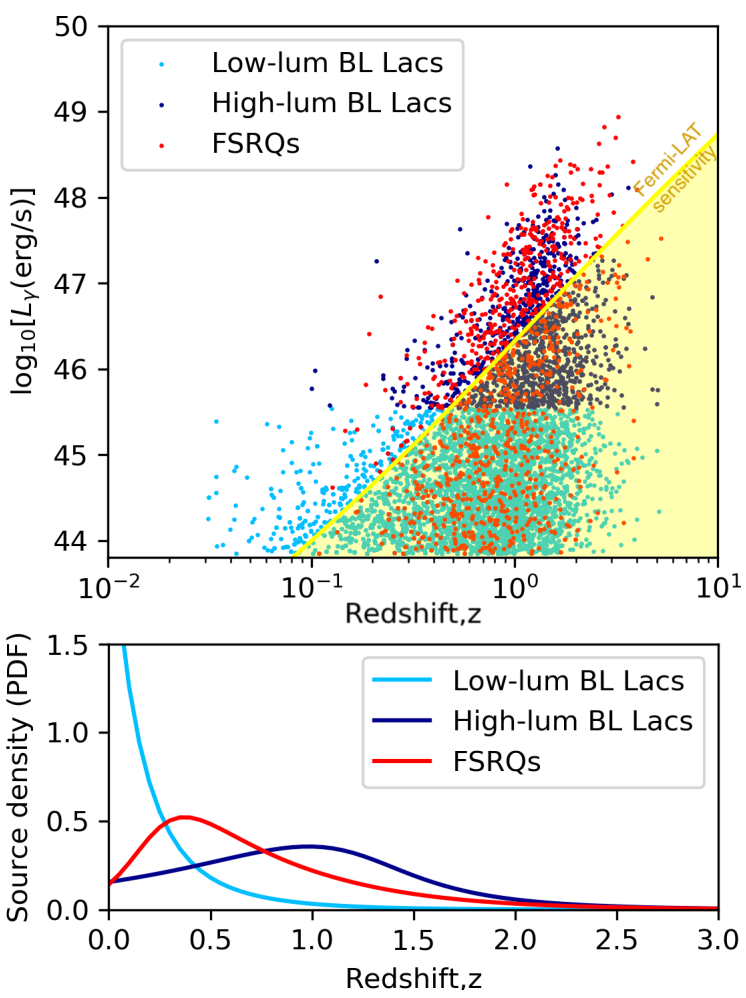

FIG. 1. Representation of the blazar population as a function of redshift and luminosity, following the model by $[65,66]$. The yellow region represents the phase space that falls below the sensitivity of the Fermi LAT $\gamma$-ray telescope. Here, we divide blazars into FSRQs, low-luminosity BL Lacs, and high-luminosity BL Lacs. The lower panel shows the same distribution in redshift (integrated over luminosity) and it clearly illustrates the strong negative evolution of low-luminosity BL Lacs compared to high-luminosity AGN (BL Lacs and FSRQs).

Their relative abundances, after acceleration and before undergoing photointeractions, follow the composition suggested in Ref. [12], namely relative abundances of 1.00 , $0.46,0.30$, and 0.14 , respectively. These fractions correspond to the Galactic cosmic-ray composition and can in fact originate from solar system abundances through chemical enhancement during the acceleration process [68]. Fine-tuning the assumed composition of the primary cosmic rays could of course improve the description of the observed UHECR data, at the cost of extra parameters; however, such detailed description is not the goal of this study. Furthermore, as discussed in the Supplemental Material [41], the conclusions regarding neutrino emission are not sensitive to this particular choice.

The maximum energies of the cosmic rays are determined self-consistently depending on the specific nuclear isotope, based on the balance between the particle's energy loss and acceleration timescales. The spectrum of nonthermal photons in the jet is adopted from the blazar sequence paradigm $[69,70]$, an assumption used in previous multimessenger studies of blazars $[23,26]$. 
In this approximation, the nonthermal photon spectrum depends only on the $\gamma$-ray luminosity $L_{\gamma}$ of the AGN.

The accelerated UHECR nuclei interact with target photons in the AGN jet, leading to photodisintegration and photopion production, which implies the emission of neutrinos with energies roughly following that of the primary cosmic rays. We simulate these radiative processes including the nuclear cascade in the source self-consistently, using the numerical code NEUCOSMA $[71,72]$ and the AGN model introduced in Ref. [26]. For BL Lacs, we implement a one-zone model where the cosmic rays interact with the nonthermal radiation produced in the jet; for FSRQs, additional photons emitted from the broad line region and the dust torus provide additional targets for the photohadronic interactions, which enhance the neutrino emission. For the extragalactic propagation of the UHECRs from the source to Earth, we use the novel numerical code PRINCE [39].

We find that in high-luminosity sources, especially in FSRQs, the highly efficient photohadronic interactions lead to abundant neutrino production and to an extensive nuclear cascade. Low-luminosity BL Lacs, on the other hand, are highly efficient UHECR emitters and inefficient neutrino emitters because of the low photon densities in the jet, which allow the accelerated UHECRs simply to escape the source without interacting - meaning that they exhibit a rigidity-dependent maximal energy as typically required in UHECR fits. Furthermore, the strong negative cosmological evolution of these sources also leads to minimal cosmicray energy losses during propagation (as shown in Fig. 1, most low-luminosity BL Lacs have a redshift $z<0.5$ ).

We have tested a wide range of values of AGN properties, such as the baryonic loading, the cosmic-ray acceleration efficiency, and the size of the radiation zone (see Sec. II of the Supplemental Material [41] where the AGN populations are discussed). We have found that not all of these parameters can be similar across all sources: at least the baryonic loading has to be higher for low-luminosity BL Lacs compared to high-luminosity AGN. The reason is that the efficient neutrino emission from high-luminosity jets would violate $\mathrm{PeV}-\mathrm{EeV}$ neutrino bounds. This means that current neutrino observations break a possible parameter degeneracy and provide evidence that AGN of different populations must have different properties if they are to
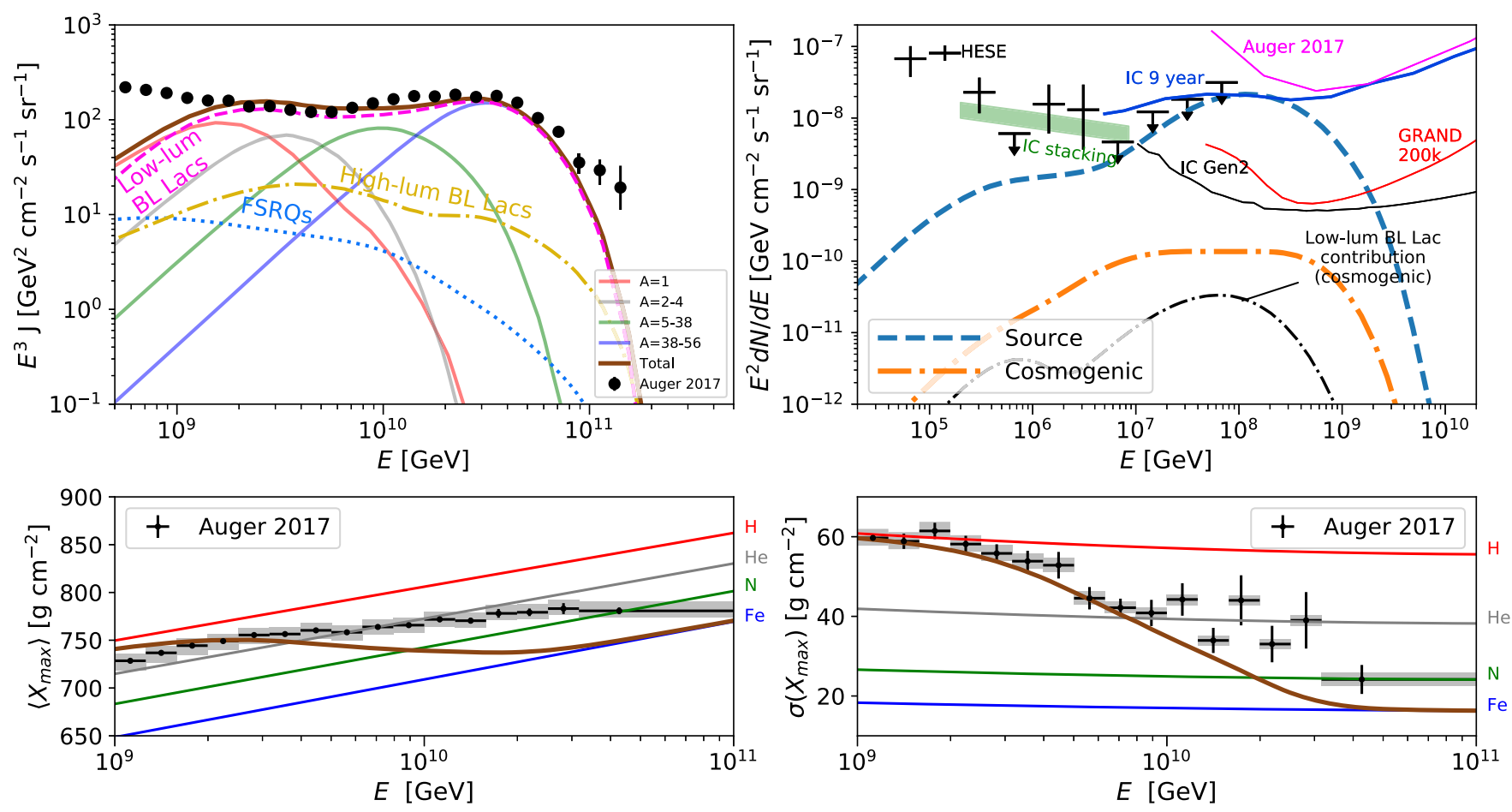

FIG. 2. Description of UHECR spectrum and composition as well as predicted neutrino fluxes. Top left: simulated UHECR spectrum from the entire AGN population (dominated by low-luminosity BL Lacs), compared to data from the Pierre Auger Observatory (Auger, [32]). Top right: maximum (all-flavor) diffuse neutrino flux (dominated by FSRQs) that can be obtained self-consistently without violating current IceCube observations, namely the flux of high-energy starting events (HESE) (black, [73]), the stacking limit for blazars assuming a spectral index of 2.2 (green band, [7]), and the upper limits up to extremely high energies (blue curve, [13]). Also shown are the sensitivity of Auger (magenta, [74]), of the future radio array of IceCube-Gen2 (olive green, [33]) and of the planned radio neutrino detector GRAND [34]. The two bottom panels show the average (bottom left) and standard deviation (bottom right) of the depth of the cosmic-ray shower maximum, $X_{\max }$, compared to Auger measurements [75]. The colored lines correspond to the values expected for different isotopes according to the Epos-LHC air-shower model [75]. 
power the UHECR flux. This may also possibly point to different cosmic-ray acceleration mechanisms in these two source classes.

We summarize our main result in Fig. 2. In the upper left panel we can see that it is possible to interpret the shape of the UHECR flux at and above the ankle with a dominant contribution from low-luminosity BL Lacs. In spite of the assumption that FSRQs have the same cosmic-ray acceleration efficiency as BL Lacs (10\%, see Supplemental Material [41]), their contribution is softer due to their large cosmological distances (dotted blue curve).

While low-luminosity BL Lacs can explain the UHECR flux, high-energy neutrinos are efficiently produced mainly in FSRQs, which dominate the spectrum shown in the upper right panel of Fig. 2. In that sense, the neutrino flux is predominantly constrained by the upper limits provided by IceCube, such as the stacking limit in the PeV range, and less so by cosmic-ray data. In fact, in this model FSRQs contribute to the UHECRs flux at a level of at most $10 \%$ at $\mathrm{EeV}$ energies, and the neutrino flux from FSRQs is therefore not guaranteed. However, because the UHECRs emitted by FSRQs have a high proton content, their contribution does improve the composition observables below $10^{10} \mathrm{GeV}$ (see lower panels). In addition, if the baryonic loading of FSRQs is to be of the same order of magnitude as that of low-luminosity BL Lacs, a high neutrino flux is more naturally expected.

While the identification of FSRQs as neutrino emitters and of BL Lacs as UHECR emitters is in agreement with the previous literature [23], we additionally conclude that the neutrinos emitted by the sources can actually outshine the overall flux of cosmogenic neutrinos. This shows that in future searches in the $\mathrm{EeV}$ range, high-energy neutrinos from FSRQs should outshine the overall cosmogenic contribution from AGN, an important result for the next generation of EeV neutrino telescopes. For example, source neutrinos point directly to the sources, which allows for different detection techniques such as stacking searches, flare analyses, or multimessenger follow-ups. On the contrary, cosmogenic neutrinos may be isotropically distributed. [In general, cosmogenic neutrinos are not necessarily isotropically distributed (see e.g., Ref. [76]). However, since the Auger results indicate that most UHECRs at the highest energies are heavy nuclei, we expect significant deflections in extragalactic magnetic fields, leading to a high level of isotropy in the cosmogenic neutrino flux.] Interestingly, the same FSRQs that may dominate the $\mathrm{EeV}$ neutrino flux may also contribute a few events at $\mathrm{PeV}$ energies.

Regarding the composition observables (lower panels of Fig. 2), the result captures the general tendency of a heavier composition with energy. However, the predicted composition at high energies is too heavy compared to Auger observations, because the proton-rich emission from FSRQs has a corresponding neutrino flux that is

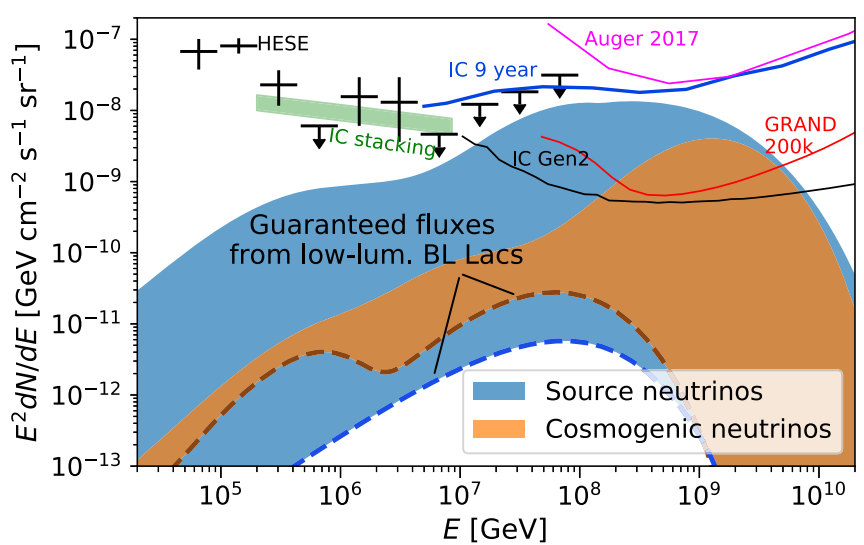

FIG. 3. Predicted (all-flavor) neutrino flux range from the entire AGN population, produced through UHECR interactions inside the sources (source neutrinos, blue region) and during extragalactic propagation (cosmogenic neutrinos, orange region). The neutrino flux can saturate current IceCube limits at EeV energies while avoiding current stacking limits at sub-PeV to $\mathrm{PeV}$ energies. This maximum flux would originate mainly in source interactions in FSRQs, with a subdominant cosmogenic contribution. At the same time, the contribution from low-luminosity BL Lacs (dashed curves), is a guaranteed minimum flux if this source class saturates the UHECR flux as shown in Fig. 2.

constrained by the current IceCube limits—as shown in the upper right panel. This discrepancy may indicate that additional parameters of the different AGN populations could be different, such as the initial cosmic-ray composition in the sources (which we fixed to a Galactic-like composition), or the acceleration efficiency (see Supplemental Material, Fig. 5 [41]).

In Fig. 3 we represent the possible ranges for source neutrinos (blue band) and cosmogenic neutrinos (brown band) inferred from our analysis. Since the cosmic-ray acceleration efficiency of FSRQs is not constrained by UHECR arguments, the bands in Fig. 3 comprehend any value up to an acceleration efficiency of $100 \%$, in order to portray the full range of possibilities for the neutrino spectrum. We find that in any scenario where FSRQs dominate the neutrino flux (including the benchmark result of Fig. 2), the source neutrinos dominate over the cosmogenic component. At the same time, if low-luminosity BL Lacs do indeed power the UHECRs, then the cosmogenic neutrinos from this source class constitute at least a guaranteed flux up to $\mathrm{EeV}$ energies (dashed curve); however, without a contribution from FSRQs, such flux would be difficult to detect with the future instruments currently proposed.

Besides the UHECR spectrum and composition and the neutrino flux, relevant constraints to this problem can also be provided by the cosmogenic $\gamma$-ray flux and the arrival directions of the UHECRs. Our main result is expected to be fully compatible with measurements and limits on these observables. For a discussion on these topics, see Secs. III and IV of the Supplemental Material [41]. 
In summary, we have performed a self-consistent description of jetted AGN as the sources of the UHECRs, including a source model treating the nuclear cascade in the sources; an UHECR transport model; and a blazar population model consistent with the extragalactic $\gamma$-ray background and the evolution of the spectral energy distribution. The acceleration model and the expected injection composition have been motivated by previous results in the literature.

We have found that low-luminosity BL Lacs can describe the shape of the UHECR spectrum and power the UHECRs, while the expected source and cosmogenic neutrino fluxes are low. In order to improve the UHECR composition observables, however, a subdominant contribution from high-luminosity AGN is required that leads to large neutrino fluxes within the reach of upcoming experiments. We have also found evidence that the fundamental physical parameters may have to be different for the different subpopulations of AGN if this source class powers the UHECRs. A possible degeneracy in these parameters, namely in the baryonic loading, is already broken by current neutrino observations. This may point toward different acceleration mechanisms at work in different AGN populations.

Our results demonstrate that it is plausible that astrophysical source neutrinos from AGN in fact outshine the cosmogenic neutrino flux, which means that cosmogenic neutrinos could actually be the background and not the foreground at $\mathrm{EeV}$ neutrino energies. Since source neutrinos can be identified and disentangled with different techniques, such as stacking searches, flare analyses, or multimessenger follow-ups, this result has profound implications for the planning and analysis of future radio-detection experiments in the EeV range, and will potentially open a new field of research. Examples are point-source or multiplet analyses, which may lead to the discovery of sources by finding anisotropies in the neutrino sky at the highest energies. Note that the source neutrino flux spans over many orders of magnitude in energy, and combined analysis between $\mathrm{TeV}-\mathrm{PeV}$ and $\mathrm{EeV}$ neutrino experiments will also be of great interest.

The authors would like to thank Anna Franckowiak and Anna Nelles for comments on the manuscript. This project has received funding from the European Research Council (ERC) under the European Union's Horizon 2020 research and innovation program (Grant No. 646623). X. R. was supported by the Initiative and Networking Fund of the Helmholtz Association.

*xavier.rodrigues@desy.de

[1] M. Ackermann et al. (Fermi-LAT Collaboration), Phys. Rev. Lett. 116, 151105 (2016).

[2] A. Aab et al. (Pierre Auger Collaboration), Astrophys. J. 853, L29 (2018).
[3] M. G. Aartsen et al. (IceCube Collaboration), Science 342, 1242856 (2013).

[4] M.G. Aartsen et al. (Liverpool Telescope, MAGIC, H.E.S.S., AGILE, Kiso, VLA/17B-403, INTEGRAL, Kapteyn, Subaru, HAWC, Fermi-LAT, ASAS-SN, VERITAS, Kanata, IceCube, and Swift NuSTAR Collaborations), Science 361, eaat1378 (2018).

[5] M. G. Aartsen et al. (IceCube Collaboration), Science 361, 147 (2018).

[6] M. Kadler et al., Nat. Phys. 12, 807 (2016).

[7] M. G. Aartsen et al. (IceCube Collaboration), Astrophys. J. 835, 45 (2017).

[8] A. Palladino, X. Rodrigues, S. Gao, and W. Winter, Astrophys. J. 871, 41 (2019).

[9] A. Neronov and D. V. Semikoz, Sov. Phys. JETP 158, 295 (2020).

[10] S. S. Kimura, K. Murase, and B. T. Zhang, Phys. Rev. D 97, 023026 (2018).

[11] J. H. Matthews, A. R. Bell, K. M. Blundell, and A. T. Araudo, Mon. Not. R. Astron. Soc. 482, 4303 (2019).

[12] R. Mbarek and D. Caprioli, Astrophys. J. 886, 8 (2019).

[13] M. G. Aartsen et al. (IceCube Collaboration), Phys. Rev. D 98, 062003 (2018).

[14] A. Aab et al. (Pierre Auger Collaboration), J. Cosmol. Astropart. Phys. 10 (2019) 022.

[15] R. J. Protheroe and A. P. Szabo, Phys. Rev. Lett. 69, 2885 (1992).

[16] K. Mannheim, Astron. Astrophys. 269, 67 (1993).

[17] D. Gorbunov, P. Tinyakov, I. Tkachev, and S. V. Troitsky, Astrophys. J. Lett. 577, L93 (2002).

[18] F. Halzen and D. Hooper, Rep. Prog. Phys. 65, 1025 (2002).

[19] C. Dermer, S. Razzaque, J. Finke, and A. Atoyan, New J. Phys. 11, 065016 (2009).

[20] W. Essey, O. E. Kalashev, A. Kusenko, and J. F. Beacom, Phys. Rev. Lett. 104, 141102 (2010).

[21] C. D. Dermer and S. Razzaque, Astrophys. J. 724, 1366 (2010).

[22] K. Murase, C. D. Dermer, H. Takami, and G. Migliori, Astrophys. J. 749, 63 (2012).

[23] K. Murase, Y. Inoue, and C. D. Dermer, Phys. Rev. D 90, 023007 (2014).

[24] E. Resconi, S. Coenders, P. Padovani, P. Giommi, and L. Caccianiga, Mon. Not. R. Astron. Soc. 468, 597 (2017).

[25] P. Padovani, E. Resconi, P. Giommi, B. Arsioli, and Y. L. Chang, Mon. Not. R. Astron. Soc. 457, 3582 (2016).

[26] X. Rodrigues, A. Fedynitch, S. Gao, D. Boncioli, and W. Winter, Astrophys. J. 854, 54 (2018).

[27] B. Eichmann, J. P. Rachen, L. Merten, A. van Vliet, and J. Becker Tjus, J. Cosmol. Astropart. Phys. 02 (2018) 036.

[28] C. Righi, A. Palladino, F. Tavecchio, and F. Vissani, Astron. Astrophys. 642, A92 (2020).

[29] K. Fang and K. Murase, Nat. Phys. 14, 396 (2018).

[30] D. Biehl, D. Boncioli, C. Lunardini, and W. Winter, Sci. Rep. 8, 10828 (2018).

[31] D. Boncioli, D. Biehl, and W. Winter, Astrophys. J. 872, 110 (2019).

[32] F. Fenu (Pierre Auger Collaboration), Proc. Sci. ICRC2017 (2018) 486.

[33] M. G. Aartsen et al. (IceCube Collaboration), arXiv:1911 .02561 . 
[34] J. Álvarez Muñiz et al. (GRAND Collaboration), Sci. China Phys. Mech. Astron. 63, 219501 (2020).

[35] P. Allison et al., Astropart. Phys. 35, 457 (2012).

[36] S. W. Barwick et al. (ARIANNA Collaboration), Astropart. Phys. 70, 12 (2015).

[37] A. Aab et al. (Pierre Auger Collaboration), J. Cosmol. Astropart. Phys. 04 (2017) 038.

[38] R. Alves Batista, R. M. de Almeida, B. Lago, and K. Kotera, J. Cosmol. Astropart. Phys. 01 (2019) 002.

[39] J. Heinze, A. Fedynitch, D. Boncioli, and W. Winter, Astrophys. J. 873, 88 (2019).

[40] A. van Vliet, R. A. Batista, and J. R. Hörandel, Phys. Rev. D 100, 021302(R) (2019).

[41] See Supplemental Material at http://link.aps.org/ supplemental/10.1103/PhysRevLett.126.191101 for an indepth description of the model properties, which includes Refs. [42-63].

[42] M. Ackermann et al. (Fermi-LAT Collaboration), Astrophys. J. 810, 14 (2015).

[43] A. J. Koning, S. Hilaire, and M. C. Duijvestijn, in Proceedings, International Conference on Nuclear Data for Science and Technology (2007), pp. 211-214.

[44] R. Alves Batista, A. Dundovic, M. Erdmann, K.-H. Kampert, D. Kuempel, G. Müller, G. Sigl, A. van Vliet, D. Walz, and T. Winchen, J. Cosmol. Astropart. Phys. 05 (2016) 038.

[45] R. Aloisio, D. Boncioli, A. Di Matteo, A. F. Grillo, S. Petrera, and F. Salamida, J. Cosmol. Astropart. Phys. 11 (2017) 009.

[46] J. L. Puget, F. W. Stecker, and J. H. Bredekamp, Astrophys. J. 205, 638 (1976).

[47] F. Riehn, R. Engel, A. Fedynitch, T. K. Gaisser, and T. Stanev, Proc. Sci. ICRC2015 (2016) 558 [arXiv:1510 .00568].

[48] D. Hooper, A. M. Taylor, and S. Sarkar, Astropart. Phys. 34, 340 (2011).

[49] E. Roulet, G. Sigl, A. van Vliet, and S. Mollerach, J. Cosmol. Astropart. Phys. 01 (2013) 028.

[50] R.-Y. Liu, A. M. Taylor, X.-Y. Wang, and F. A. Aharonian, Phys. Rev. D 94, 043008 (2016).

[51] A. van Vliet, EPJ Web Conf. 135, 03001 (2017).

[52] A. D. Supanitsky, Phys. Rev. D 94, 063002 (2016).

[53] V. Berezinsky, A. Gazizov, and O. Kalashev, Astropart. Phys. 84, 52 (2016).
[54] A. Aab et al. (Pierre Auger Collaboration), Science 357, 1266 (2017).

[55] A. Aab et al. (Pierre Auger Collaboration), Astrophys. J. 868, 4 (2018).

[56] L. Caccianiga et al. (Pierre Auger Collaboration), Proc. Sci. ICRC2019 (2019) 206.

[57] J. H. Matthews, A. R. Bell, K. M. Blundell, and A. T. Araudo, Mon. Not. R. Astron. Soc.: Lett. 479, L76 (2018).

[58] G. E. Romero, J. A. Combi, L. A. Anchordoqui, and S. Perez Bergliaffa, Astropart. Phys. 5, 279 (1996).

[59] P. L. Biermann and V. de Souza, Astrophys. J. 746, 72 (2012).

[60] G. R. Farrar and M. S. Sutherland, J. Cosmol. Astropart. Phys. 05 (2019) 004.

[61] S. Razzaque, C. D. Dermer, and J. D. Finke, Astrophys. J. 745, 196 (2012).

[62] H. Takami, K. Murase, and C. D. Dermer, Astrophys. J. 817, 59 (2016).

[63] R. Alves Batista, M.-S. Shin, J. Devriendt, D. Semikoz, and G. Sigl, Phys. Rev. D 96, 023010 (2017).

[64] C. M. Urry and P. Padovani, Publ. Astron. Soc. Pac. 107, 803 (1995).

[65] M. Ajello et al., Astrophys. J. 751, 108 (2012).

[66] M. Ajello et al., Astrophys. J. 780, 73 (2014).

[67] A. Abdo et al. (Fermi-LAT Collaboration), Phys. Rev. Lett. 104, 101101 (2010).

[68] D. Caprioli, D. T. Yi, and A. Spitkovsky, Phys. Rev. Lett. 119, 171101 (2017).

[69] G. Fossati, L. Maraschi, A. Celotti, A. Comastri, and G. Ghisellini, Mon. Not. R. Astron. Soc. 299, 433 (1998).

[70] G. Ghisellini, C. Righi, L. Costamante, and F. Tavecchio, Mon. Not. R. Astron. Soc. 469, 255 (2017).

[71] S. Hümmer, M. Rüger, F. Spanier, and W. Winter, Astrophys. J. 721, 630 (2010).

[72] P. Baerwald, S. Hümmer, and W. Winter, Phys. Rev. D 83, 067303 (2011).

[73] M. G. Aartsen et al. (IceCube Collaboration), Astrophys. J. 809, 98 (2015).

[74] E. Zas (Pierre Auger Collaboration), Proc. Sci. ICRC2017 (2018) 972.

[75] J. Bellido (Pierre Auger Collaboration), Proc. Sci. ICRC2017 (2018) 506.

[76] W. Essey, O. Kalashev, A. Kusenko, and J. F. Beacom, Astrophys. J. 731, 51 (2011). 